\title{
Telefonischer Kontakt
}

\section{BAG haben Spielraum beim Honorar}

\author{
Die telefonische Beratung von Patienten nach EBM-Nummer 01435 \\ kann nicht neben der Versichertenpauschale vergütet werden. Für eine \\ Berufsausübungsgemeinschaft (BAG) gibt es aber Spielraum bei der \\ Abrechnung, weil die Position an den Arztfall gebunden ist.
}

$\mathrm{D}$ ie Abrechnung der ärztlichen Leistung nach Gebührenordnungsposition (GOP) 01435, der haus-/fachärztlichen Bereitschaftspauschale, bereitet vielen Praxen Schwierigkeiten. Im Mittelpunkt steht dabei vor allen immer wieder die Frage, wann genau diese GOP berechnet werden kann - und wann eben nicht.

Um die richtige Antwort zu finden, müssen sich die abrechnenden Ärzte den obligaten Leistungsinhalt im Zusammenhang mit den Anmerkungen und den Allgemeinen Bestimmungen im EBM genau anschauen.

Gefordert ist bei der betreffenden Position die „telefonische Beratung des $\mathrm{Pa}$ tienten im Zusammenhang mit einer Erkrankung durch den Arzt bei Kontaktaufnahme durch den Patienten und/oder ein anderer mittelbarer Kontakt gemäß 4.3.1 der Allgemeinen Bestimmungen.“

Die EBM-Nummer 01435 ist demzufolge dann berechnungsfähig, wenn eine telefonische Beratung des Patienten durch den Arzt stattfindet. Dabei muss der Patient den Arzt oder die Praxis angerufen haben. Das ist beispielsweise dann der Fall, wenn der Patient sich etwa nach Laborbefunden oder dem Ergebnis einer histologischen Untersuchung erkundigt und der Arzt im Zuge dieser Auskunft auch beratend tätig wird.

\section{Wichtige Frage: Wurde die} Leistung im Vorquartal erbracht? Wichtig ist, dass die GOP 01435 im Arztfall laut den Anmerkungen im EBM nicht neben einer Versicherten- oder Grundpauschale berechnet werden darf. Sie kommt in Einzelpraxen daher nur sehr selten vor. Es gibt aber Sonderfälle, in denen Patienten zu Beginn eines Quartals etwa telefonisch Befunde abfragen, die noch im Vorquartal veranlasst wurden, sodass die Patienten in der Konsequenz dann im laufenden Quartal

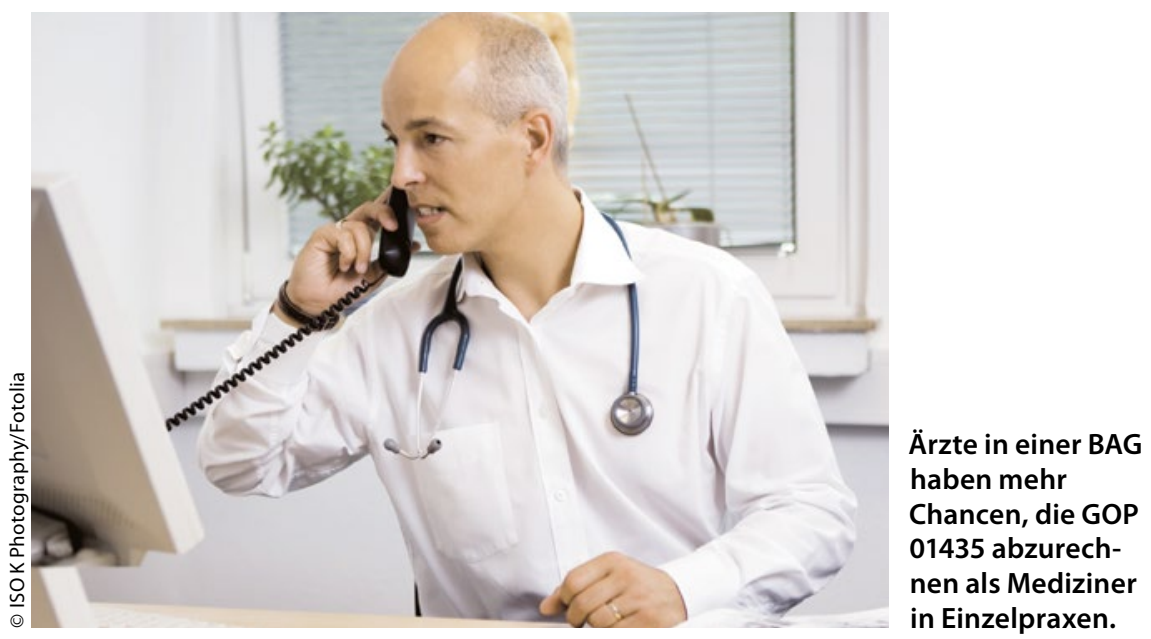

nicht mehr persönlich in die Praxis kommen müssen. In diesen Fällen sollten Ärzte nicht vergessen, die Leistung für die telefonische Beratung anzusetzen. In einer Gemeinschaftspraxis oder einer BAG kann die GOP 01435 ebenfalls für eine alleinige telefonische Beratung des Patienten im Quartal berechnet werden.

Daraus folgt: In einer Einzelpraxis kann die EBM-Nummer 01435 nur dann abgerechnet werden, wenn in dem entsprechenden Quartal ausschließlich eine telefonische oder eine mittelbare Beratung stattgefunden hat. In einer BAG ist bei der Berechnung der GOP 01435 hingegen etwas mehr Luft für Ärzte, wenn sie die Leistung abrechnen möchten.

\section{Teamwork macht sich bezahlt}

Vorteil für die BAG im Zusammenhang mit der telefonischen Beratung: Kommt es in einer Einrichtung, in der bei einem Patienten bereits ein persönlicher Kontakt mit Arzt A erfolgt ist und daher die Versichertenpauschale abgerechnet werden kann, zu einem telefonischen Kontakt mit Arzt B, kann dieser unter seiner lebenslangen Arztnummer (LANR) die Position 01435 dafür abrechnen.

Wird eine weitere Beratung von einem weiteren Arzt C der BAG durchgeführt, der nicht die Versichertenpauschale abgerechnet hat, kann dieser Kollege ebenfalls die Position ansetzen, weil damit wieder ein neuer Arztfall entsteht. Das wiederum hat noch weitere positive Auswirkungen für die Praxis, denn jeder Arztfall ist wichtig für die Berechnung des Regelleistungsvolumens (RLV), weil jeder zusätzliche Arztfall das RLV einer Praxis im selben Quartal des Folgejahres erhöht.

Es gilt daher in einer BAG, jeden Kontakt von Patienten mit weiteren Ärzten in der Abrechnung mit der KV sichtbar zu machen, zum Beispiel über die GOP 01435, wenn diese Ziffer möglich ist.

Peter Schlüter 